with multiple sexual partners, persistent/recurrent infection, and potential antimicrobial resistance; however, none of the HCP believed there was a difference in efficacy based on a client's sexual preferences or gender identity. HCP who provided EPT described strategies to overcome these barriers, such as capitalizing on pharmacies and phone calls or videoconference calls with patients' partners to discuss potential allergies, treatment regimens, and ensuring linkage to HIV testing. HCP described how telemedicine and the use of peer health navigators could help overcome systemic barriers to regular STI testing (e.g., transportation, delayed appointment times) and potentially overcome medical mistrust around HIV prevention and care.

Conclusion The provision of EPT represents an overlooked yet important strategy to curb increasing STI rates among MSM and TW. Changing EPT legislation and CDC guidelines to include MSM and TW represents a promising avenue to link these communities to HIV and other STI prevention services. Disclosure No significant relationships.

\section{P507 DISCLOSURE OF SAME-SEX BEHAVIOR AND EXTRAGENITAL STI TESTING AMONG MEN WHO HAVE SEX WITH MEN - AMERICAN MEN'S INTERNET SURVEY}

${ }^{1}$ Alex De Voux* ${ }^{2}$ Kyle Bernstein, ${ }^{2}$ Robert Kirkcaldy, ${ }^{3}$ Maria Zlotorzynska, ${ }^{3}$ Travis Sanchez. ${ }^{1}$ CDC, Division of STD Prevention, Atlanta, USA; ${ }^{2}$ Centers for Disease Control and Prevention, Atlanta, USA; ${ }^{3}$ Emory University Rollins School of Public Health, Department of Epidemiology, Atlanta, USA

\subsection{6/sextrans-2019-sti.587}

Background Current guidelines recommend that sexually active men who have sex with men (MSM) be screened at least annually for bacterial sexually transmitted infections (STI) at sites of sexual contact regardless of condom use. Extragenital (rectal and pharyngeal) STIs, common among MSM, are mostly asymptomatic and often missed with urethral-only screening. Disclosure of same-sex behaviors by MSM to healthcare providers (HCP) or HCP elicitation of same-sex behaviors through sexual behavioral assessments may facilitate appropriate extragenital STI testing. We describe self-reported prevalence of extragenital STI testing in the past 12 months (p12m) among MSM in the United States stratified by disclosure of same-sex behavior.

Methods Data were obtained from the 2017 American Men's Internet Survey (AMIS), an annual cross-sectional behavioral internet survey of MSM in the United States. We examined the prevalence of MSM who reported any STI test and an extragenital STI test in the $\mathrm{p} 12 \mathrm{~m}$ stratified by disclosure of same-sex behavior among HIV-positive and HIV-negative MSM.

Results Of 10, 049 sexually-active MSM, 42\% reported any STI test and $16 \%$ reported an extragenital STI test in the p12m. Among HIV-negative MSM, 21\% reported an extragenital STI test in p12m; among HIV-positive MSM, 32\% reported an extragenital STI test in the p12m. Ever disclosing same-sex behavior was associated with a higher prevalence of extragenital STI testing $(\mathrm{PR}=1.68 ; 95 \%$ CI 1.53-1.86) among HIV-negative MSM, but not among HIV-positive MSM $(\mathrm{PR}=1.01 ; 95 \%$ CI 0.81-1.25).

Conclusion The prevalence of any STI testing and extragenital STI testing in the $\mathrm{p} 12 \mathrm{~m}$ among MSM was low. To implement STI recommendations, HCPs must be aware of their patients' sexual behaviors. Disclosure of same-sex behaviors by MSM to their HCP or elicitation of these behaviors by HCPs through sexual behavioral assessments could increase STI testing, particularly at relevant anatomic sites of exposure.

Disclosure No significant relationships.

\section{P508 HEPATITIS C REINFECTION RATES AFTER CURE OR CLEARANCE AMONG HIV-INFECTED AND UNINFECTED MEN WHO HAVE SEX WITH MEN}

Carmine Rossi, Zahid Butt, Maryam Darvishian*, Stanley Wong, Amanda Yu, Maria Alvarez, Mel Krajden, Naveed Janjua. BC CDC, Vancouver, Canada

\subsection{6/sextrans-2019-sti.588}

Background Increasing rates hepatitis $\mathrm{C}$ virus (HCV) infection associated with ongoing risk activity have been reported after successful cure or viral clearance. We assessed factors associated with reinfection after treatment-induced or spontaneous clearance (SC) in both HIV-infected and uninfected men who have sex with men (MSM) in British Columbia.

Methods We followed HIV-infected and uninfected MSM who achieved sustained virologic response (SVR) to HCV treatment or had SC with $\geq 1$ subsequent HCV RNA measurement in the British Columbia Hepatitis Testers Cohort. Crude reinfection rates per 100 person-years (PYs) were calculated. Cox regression was used to model adjusted hazard ratios (HRs) and $95 \%$ confidence intervals (CI) for reinfection.

Results We identified 1,349 HCV-infected MSM with SVR $(n=856)$ or SC $(n=493)$, of which $349(26 \%)$ were HIV-positive. HIV-infected MSM were more likely to have histories of injection drug use (41\% vs $21 \%$ ), alcohol use (22\% vs $14 \%$ ) and mental health disorders ( $47 \%$ vs $28 \%$ ), compared to HIV uninfected. A total of 98 reinfections were identified, yielding an overall reinfection rate of 1.9 per 100 PY (1.0 for SVR patients and 2.7 for SC). HIV-infected MSM had higher rates of reinfection (3.1 vs 1.6 per 100 PY) than HIV uninfected individuals. In multivariable analysis, age $<35$ years (HR 3.1, 95\% CI: $1.2,8.1)$, cure through SVR(HR 0.2, 95\% CI: 0.1 , 0.4), HIV infection (HR 2.0, 95\% CI: 1.3, 3.1), problematic alcohol use (HR 2.0, 95\% CI: 1.2, 3.3), injection drug use (HR 2.7, 95\% CI: 1.6, 4.3) and mental health counseling (HR 0.2, 95\% CI: $0.1,0.4$ ) were independently associated with reinfection. Among HIV-infected, injection drug use (HR 1.9, 95\% CI: $0.8,4.2$ ) was less strongly associated with reinfection.

Conclusion Rates of HCV reinfection remain elevated among HIV-infected and uninfected MSM. Substance use is driving reinfection among HIV-negative MSM, while sexual transmission may be more important among HIV-positive MSM.

Disclosure No significant relationships. 